\title{
A SURVEY ON ENERGY EFFICIENT WITH TASK CONSOLIDATION IN THE VIRTUALIZED CLOUD COMPUTING ENVIRONMENT
}

\author{
Elizabeth Sylvester Mkoba ${ }^{1}$, Mokhtar Abdullah Abdo Saif ${ }^{2}$ \\ ${ }^{1,2}$ Student, School of Computer Science and Technology, Dalian University of Technology, Liaoning, China
}

\begin{abstract}
Cloud computing is a new model of computing that is widely used in today's industry, organizations and society in information technology service delivery as a utility. It enables organizations to reduce operational expenditure and capital expenditure. However, cloud computing with underutilized resources still consumes an unacceptable amount of energy than fully utilized resource. Many techniques for optimizing energy consumption in virtualized cloud have been proposed. This paper surveys different energy efficient models with task consolidation in the virtualized cloud computing environment.
\end{abstract}

Keywords: Cloud computing, Virtualization, Task consolidation, Energy consumption, Virtual machine

\section{INTRODUCTION}

Cloud computing is a new service model for Information Technology provision, often based on virtualization and distributed computing technologies. Resource provisioning based on virtual machine (VM) has been widely accepted and adopted in cloud computing environments. Virtualized resources with a low utilization rate still consume an unacceptable amount of energy compared to the energy consumption of a fully utilized cloud computing. According to [13], energy consumption of an idle resource can be as much as $60 \%$ or peak power. In cloud computing, there is a relationship between energy consumption and resource utilization. Task consolidation is an effective technique greatly enabled by virtualization technologies, which facilitate the concurrent execution of several tasks, maximize resource utilization and in turn reduce the energy consumption [1].

The cloud resource utilization can be realized by dynamically allocating resources based on utilization analysis and prediction [5]. A good resource management scheme is fundamental for on-demand resource allocation, performance optimization, load balancing and energy saving in the cloud system. Although some existing task consolidation algorithms have been developed to address the energy efficiency problem, they normally result in high migration costs, ignoring other virtual resources which influence energy consumption as well as low resource utilization due to ignoring the multidimensional characteristics of virtual machines.

In this survey paper, energy optimization techniques are discussed. The rest of the paper is organized as follows: Section 2 presents task consolidation overview, Section 3 presents the literature review of the existing methods of energy efficient with task consolidation, and Section 4 presents a conclusion.

\section{TASK CONSOLIDATION}

\subsection{Task Consolidation Problem}

The task consolidation problem is the process of assigning a set of $t$ tasks (service requests) to a set of $r$ cloud computing resources in order to maximize resource utilization and ultimately to minimize energy consumption. Time constraints are directly related to the resource usage associated with the tasks. In the task consolidation problem, the resource allocated to a particular task must sufficiently provide the resource usage of the given task. Task consolidation aims at effective usage of cloud resources by consolidating a set of tasks into a small number of virtual machines. The advantage of effective resource usage is to reduce monetary cost by reducing: (a) amount of virtual machines; (b) labors required to maintain virtual machines; (c) floor space; and (d) energy consumption.

\subsection{Task Parameters}

There is a relationship between energy consumption, system configurations, performance and task workload in the virtual machines. When tasks are running in the virtual machines resources are utilized such as CPU, memory, disk, network bandwidth which lead to energy consumption. The workload of a task comprises with: (a) data size for the task; (b) number of processes for the task; (c) size of data to be transmitted (d) task processing time. The energy consumption is highly influenced by the workloads for a task because they consume resources, as shown in figure 1 . The design of the cloud computing system needs to take into account how the following tasks categories are executed in the virtual machines: (a) communication-intensive tasks; (b) computation-intensive tasks; (c) data-intensive tasks. Furthermore, during the cloud system design these questions need to answered such as what are the task characteristics?, 
when to migrate the tasks?, which task to migrate? how to reduce the tasks migration overheads?

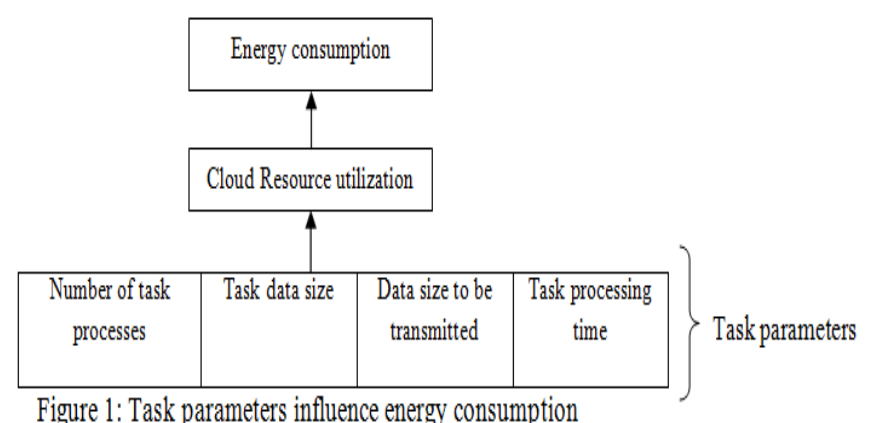

\section{EXISTING ENERGY EFFICIENT MODELS WITH TASK ONSOLIDATION IN THE CLOUDS}

\subsection{Energy Conscious Task Consolidation}

The model proposed by [13] for task consolidation is called energy-conscious task consolidation (ECTC). ECTC computes the energy consumption of a given task on a selected resource and ECTC cost function is designed to encourage resource sharing.

However, this model does not take into consideration other resources which have influence in energy consumption such as disk utilization, memory utilization, network bandwidth usage, application software. It tends to increase number of virtual machines within the cluster. It does not consider the migration of task from one virtual cluster to another.

\subsection{Maximum Rate Utilization of Resources}

In [13], authors studied energy efficient utilization of resource in the cloud computing systems. The problem addressed is a resource allocation strategy that takes into account resource utilization that leads to better energy efficiency. The cloud computing system utilizes virtualization technologies where tasks can be easily consolidated which is an effective method to increase resource utilization and in turn reduces energy consumption. They proposed task consolidation Maximum rate Utilization (MaxUtil). MaxUtil looks after the more energy efficient resources in terms of resource utilization. The energy model is devised on the basis that processor utilization has a linear relationship with energy consumption.

The task consolidation problem is modeled as a bin packing problem where virtual machines represent bins, tasks are objects to be packed in the bins and CPU resource utilization represents bin dimension. They proposed $100 \%$ resource utilization rule for virtual machines and makes task consolidation decision based on the resource utilization which is a key indicator for energy efficiency. The advantage of this method is to reduce number of virtual machines in the cluster which resulted in minimizing energy consumption in the cloud computing system.

However, the deficiencies of the proposed MaxUtil model are: (a) it assumes energy consumption is linear to resource utilization focused on CPU usage without considering other resources utilization such as disk, network bandwidth, memory etc.; (b) the incorporation of task migration increased energy consumption because migrated tasks tend to be with short remaining processing time and these tasks are most likely to hinder the consolidation of a new arriving task (c) the migration of task from one virtual cluster to another is not modeled.

\subsection{Energy Task Consolidation}

The authors [1] proposed energy-aware task consolidation (ETC) technique to optimize energy consumption of virtual clusters. The CPU and network utilization are taken into account when dealing with task migration in the virtual clusters. They proposed $70 \%$ CPU utilization principle to manage task consolidation among virtual clusters in the cloud computing system. When the CPU utilization in a virtual cluster is above $70 \%$, the task is migrated to another cluster. If there are multiple virtual machines in the clusters available to receive the tasks, the one with minimal energy cost is chosen to perform task consolidation.

Their experiment results show that energy-aware task consolidation technique can reduce power consumption in managing task consolidation for cloud systems. However, by using $70 \%$ principle for CPU utilization, the virtual machine still consumes energy because it is not fully utilized. ETC increases number of virtual machines during the task consolidation as compared to MaxUtil technique.

\subsection{Task-Based Energy Consumption}

The authors in [11] studied task-based energy consumption in the virtualized cloud computing systems. High energy consumption directly contributes to operational costs and environmental damage such as high carbon emission. In order to address the problem, they proposed an energy consumption model to calculate the energy consumption of specific types of tasks in cloud systems and use the model to dynamically organize cloud application load. They divided tasks into three types: computation-intensive, data-intensive, and communication-intensive and measure the performance with its corresponding energy consumption.

They investigated the relationship between energy consumption, system configurations and cloud system performance. The total energy consumption of a cloud workload is divided into a fixed part and dynamic part. The proposed technique determines the energy consumption for computation-intensive, data-intensive and communicationintensive and compares it with system performance. The task 
parameters such as data size to be processed and transmitted as well as number of processes have impact on the task energy consumption model. They conducted experiments with varying system configurations and workloads with corresponding energy consumption and performance data. Their results revealed that, there is correlation coefficient of energy consumption, system configuration, and workload as well as system performance in the cloud systems.

\section{COMPARISON OF THE EXISTING METHODS}

In this survey, we compare the existing methods of energy optimization with task consolidation as shown in table 1.

\begin{tabular}{|c|c|c|c|}
\hline $\begin{array}{c}\text { Task } \\
\text { consoli- } \\
\text { dation } \\
\text { method }\end{array}$ & $\begin{array}{c}\text { System } \\
\text { resource } \\
\text { used }\end{array}$ & $\begin{array}{c}\text { Power } \\
\text { saving } \\
\text { technique }\end{array}$ & $\begin{array}{l}\text { Deficiencies } \\
\text { of the method }\end{array}$ \\
\hline ECTC & $\mathrm{CPU}$ & $\begin{array}{l}\text { Task } \\
\text { consolidati } \\
\text { on }\end{array}$ & $\begin{array}{l}\text { (a) increase number of } \\
\text { virtual machines } \\
\text { (b) has not considered } \\
\text { migration of task from } \\
\text { one cluster to another. }\end{array}$ \\
\hline MaxUtil & $\mathrm{CPU}$ & $\begin{array}{l}\text { Maximize } \\
\text { resource } \\
\text { utilization } \\
\text { to reduce } \\
\text { number of } \\
\text { virtual } \\
\text { machine } \\
\end{array}$ & $\begin{array}{l}\text { (a)has not considered } \\
\text { migration of task from } \\
\text { one cluster to another. } \\
\text { (b)has not considered } \\
\text { other resources which } \\
\text { have influence in } \\
\text { energy consumption. }\end{array}$ \\
\hline ETC & $\begin{array}{l}\text { CPU, } \\
\text { net-work }\end{array}$ & $\begin{array}{l}\text { Decrease } \\
\text { resource } \\
\text { utilization }\end{array}$ & $\begin{array}{l}\text { (a) } 70 \% \text { principle for } \\
\text { CPU utilization, the } \\
\text { virtual machine still } \\
\text { consumes energy } \\
\text { because it is not fully } \\
\text { utilized. } \\
\text { (b)ETC increases } \\
\text { number of virtual } \\
\text { machines during the } \\
\text { task consolidation as } \\
\text { compared to MaxUtil } \\
\text { technique. }\end{array}$ \\
\hline $\begin{array}{l}\text { Task- } \\
\text { based } \\
\text { energy } \\
\text { consum } \\
\text { p-tion }\end{array}$ & $\begin{array}{l}\text { CPU, } \\
\text { disk, } \\
\text { memory }\end{array}$ & $\begin{array}{l}\text { System } \\
\text { configurati } \\
\text { ons }\end{array}$ & $\begin{array}{l}\text { (a)has not considered } \\
\text { the energy } \\
\text { consumption when } \\
\text { task migrate to other } \\
\text { virtual cluster. } \\
\text { (b) has not compared } \\
\text { task resource } \\
\text { utilization against } \\
\text { energy consumption as } \\
\text { in ETC and MaxUtil } \\
\text { method. }\end{array}$ \\
\hline
\end{tabular}

\section{CONCLUSIONS}

Task consolidation is an effective technique greatly enabled by virtualization technologies, which facilitate the concurrent execution of several tasks in virtual machines and in turn reduce the energy consumption. Task consolidation in cloud computing presents a significant opportunity for energy optimization. In this paper we surveyed various existing task consolidation methods to minimize energy consumption in the virtualized cloud computing environments. The main objective of cloud providers is to develop resource provisioning and management solutions that minimize energy consumption while guaranteeing Service level Agreements. Thus, this survey can be used to improve the energy consumption models by designing energy optimization models, energy consumption monitors and energy prediction models for the cloud systems.

\section{REFERENCES}

[1] C. Hsu, S.Chen, C.Lee, H. Chang, K. Lai, K. Li and C.Rong, " Energy - Aware Task consolidation Technique for cloud computing", IEEE , CloudCom 2011, pp.115-121

[2] B.Guenter, N.Jain and C. Williams,“ Managing cost, Performance and Reliability Tradeoffs for EnergyAware Server Provisioning", IEEE INFOCOM 2011, pp.1332-1340.

[3] C. Yang, K.Wang, H. Cheng, C. Kuo and W. Chu, "Green Power Management with Dynamic Resource Allocation for cloud virtual machines", IEEE International Conference on High Performance Computing and Communications ,2011, pp.726-733

[4] R. Buyya, S.Garg and R. Calheiros, "SLA-Oriented Resource Provisioning of cloud computing: Challenges, Architecture and Solutions", IEEE International Conference on Cloud and Service Computing, 2011, pp1-10

[5] Y.Shi, X.Jiang and K. Ye, "An Energy-Efficient scheme for cloud resource provisioning based on CloudSim", IEEE International Conference on Cluster Computing, 2011, pp.595-599

[6] S.Garg, C.Yeo and R. Buyya, "Green cloud framework for improving carbon efficiency of clouds", ACM 17th International Conference, Euro-Par Parallel Processing 2011, pp.491-502

[7] F. Wuhib, R. Stadler and H. Lindgren, "Dynamic Allocation with Management - Implementation for an OpenStack Cloud", 8th International Conference on Network and Service Management 2012, pp.309-315.

[8] Z. Zhou, F. Liu, H.Jin, B.Li, B.Li and H. Jiang “, On Arbitrating the Power-Performance and Tradeoff in Saas Clouds", INFOCOM 2013, pp.872-880

[9] A. Beloglazov and R. Buyya , "Managing Overloaded Hosts for Dynamic Consolidation of Virtual Machines in Cloud Data Centers under Quality of Service Constraints". IEEE Transactions on Parallel and Distributed Systems, vol. 24, N0.7, July 2013. 
[10] P.Leelipushpam and J. Sharmila, "Live Migration Techniques in cloud environment -Survey, IEEE Conference on Information and Communication Technologies, 2013, pp.408-413.

[11] F.Chen, J. Grundy, Y.Yang, J. Schneider and Q.He, "Experimental Analysis of Task-based Energy consumption in cloud computing systems", ACM ICPE 2013, pp.295-306.

[12] L. He, D. Zou, Z. Zhang, K. Yang, H. Jin, and S. A. Jarvis, "Optimizing Resource Consumptions in Clouds",12th IEEE/ACM International Conference on Grid Computing (GRID), 2011, pp. 42-49.

[13] Lee and A.Zomaya, "Energy efficient utilization of resources in cloud computing systems", Journal of Supercomputing 2012, pp. 268-280.

\section{BIOGRAPHIES}

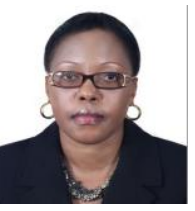

Elizabeth Sylvester Mkoba is pursuing $\mathrm{PhD}$ in Computer Science and Technology from Dalian University of Technology, China. Her research area is Cloud Computing.

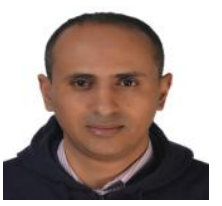

Mokhtar Abdullah Abdo Saif is pursuing $\mathrm{PhD}$ in Computer Science and Technology from Dalian University of Technology, China. His research area is Cloud Computing. 